(NASA-CR-141061). INDIRECT SYNTHESIS OF DULTI-DEGREE OF FREEDOM TRANSIENT SYSTEMS

Final Report (Virginia univ.) $25 \mathrm{p}$ HC $\$ 3.25$

\title{
INDIRECT SYNTHESIS OF
}

MULTI-DEGREE OF FREEDON

TRANSIENT SYSTEMS

Final Report on

NASA Grant No. NGR 47-005-145

Supplement No. 3

Submitted by:

Walter D. Pilkey

and

Y. H. Chen

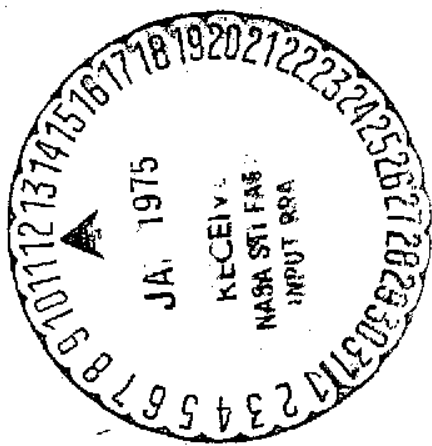

\section{SCHOOL OF ENGINEERING AND}

\section{APPLIED SCIENCE}

RESEARCH LABORATORIES FOR THE ENGINEERING SEIENCES

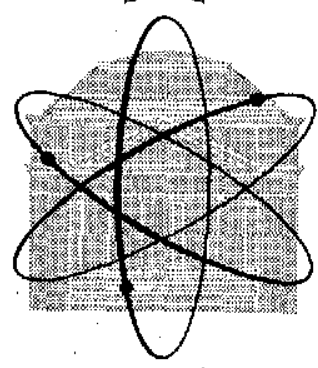

UNIVERSITY OF VIRGINIA

CHARLOTTESVILLE, VIRGINIA 22901

Report No. ESS-4085-110-74

September 1974 


\section{RESEARCH LABORATORIES FOR THE ENGINEERING SCIENCES}

The School of Engineering and Applied Science of the University of Virginia has long believed that strong research capabilities go hand in hand with effective teaching. Early in the development of its graduate training program, the School also recognized that men and women engaged in research should be as free as possible of the administrative chores involved in sponsored research. In 1959, therefore, the Research Laboratories for the Engineering Sciences (RLES) was established and assigned the administrative responsibility for such research within the School.

Currently, approximately 60 members of the faculty, who also teach at the undergraduate and graduate levels, and 30 additional professional engineers and scientists, whose primary responsibility is research, generate and conduct the investigations that make up a vigorous and wide-ranging program. The Director of RLES, a faculty member and active researcher himself, maintains familiarity with the support requirements of all research under way. He is aided by an RLES Academic Advisory Committee made up of one faculty representative from each academic department of the School. This Committee serves to inform RLES of the needs and perspectives of the research community.

In addition to administrative support, RLES is charged with providing technical assistance where it is needed. Because it is not practical for each department of the School to become self-sufficient in all phases of the supporting technology essential to present-day research, RLES makes services available through the following support groups: Machine Shop, Instrumentation, Facilities Services, Publications (including photographic facilities), and Computer Terminal Maintenance.

$\because \quad$ The purpose of RLES, then, is to provide administrative and technical assistance for sponsored fesearch carried out within the School of Engineering and Applied Science of the University of Virginia. Such research has played an important part in the University's contribution to scientific knowledge and service to the community and continues the successful partnership of University, government, and industry.

For information on current programs and capabilities, write to Director, Research Laboratories for the Engineering Sciences, Thornton Hall, University of Virginia, Charlottesville, Virginia 22901. 
INDIRECT SYNTHESIS OF

MULTI-DEGREE OF FREEDOM

TRANSIENT SYSTEMS

Final Report on

NASA Grant No. NGR 47-005-145

Supplement No. 3

Submitted by:

Walter D. Płlkey

and

Y. H. Chen

\author{
Department of Engineering Science and Systems \\ Research Laboratories for Engineering Sciences \\ University of Virginia \\ Charlottesville, Virginia
}

Report No. ESS-4085-110-74

September, 1974 


\section{Forward}

The research described in this report (University of Virginia Project No. 4085-2320) was performed at the University of Virginia Research Laboratories for the Engineering Sciences. W. D. Pilkey was principal investigator. The project was performed under NASA Grant No. NGR-47-005-145, Supplement No. 3 and administered at NASA Langley Research Center. This report covers some of the work performed during the fourth year of this grant. The valuable assistance of the technical monitor for this study; J. Sewall of NASA/ Langley, is appreciated. 


\section{Abstract}

It is shown that an indirect synthesis method can be used in the efficient optimal design of multi-degree of freedom, multi-design element, nonlinear, transient systems. The technique begins with a limiting performance analysis which requires linear programming for a kinematically linear system, following which the system is selected using system identification methods such that the designed system responds as closely as possible to the limiting performance. The efficiency is a result of the method avoiding the repetitive systems analyses accompanying other numerical optimization methods. 


\section{Introduction}

An essential ingredient of the conventional methods in the optimum design of dynamic systems has been an iterative technique that progressively searches for the open design parameters which optimize some index of system performance without violating the imposed constraints. While this search procedure presents no serious problems for simple systems with few design parameters, its practicality diminishes rapidly with increasing system complexity, and becomes formidable in terms of computational requirements for a large, real world system. The main difficulty therein stems from the fact that for each iteration, the entire system dynamics must be solved and that convergence to a global minimum, if it can be reached, usually requires a large number of iterations.

Recently a new approach called the "Indirect synthesis method" has been suggested (Ref. 1) and successfully demonstrated for a single degree of freedom system. Satisfactory results have also been obtained by applying this approach to a nonlinear impact absorber (Ref, 2) and to vehicle suspension systems (Ref. 3). The basis of the new approach is to select the open design parameters such that the response of the portion of the system being designed approximates the limiting performance solution which is independent of the hardware devices used. It suffices to solve the system dynamics only once. As a consequence, the new approach offers great computational advantages over the conventional methods. Also, by carrying out the limiting performance solution for several constraint levels it yields the limfting performance characteristics 
(trade-off curve) of the system under considexation. Apart from its use in automated design, this information, which is not avallable from conventional sources, is of considerable value to the mechanical designer in providing the feasibility characteristics of his proposed design.

Details of the formulation of the new design approach are presented in Ref 1. Essentially the method consists of two distinct phases: time optimal synthesis and parameter identification. One first replaces the design elements, regardless of configuration, by generic or control forces $u(t)$. The limiting performance solution of the resulting system is sought in the time domain of interest, yielding the optimal (1deal) generic forces $\mathrm{u}^{*}(t)$ and other ideal system responses. In the second phase of the design one selects, element by element, the design parameters that réspond most closely (in an optimal sense) to the limiting performance solution. The elements being designed can be linear, nonlinear, passive or active. The limiting performance problem is particularly simple when those portions of the system not being designed and the overal1 dynamics are linear, for then the problem can be formulated as one of linear programming. The element by element parameter design can be achieved by unconstrained curve fitting techniques such as least square or min-max fits. Thus the nonlinear programming optimization problem normally encountered in the direct methods is now replaced by a more efficient linear programming problem.

As stated earlier, the new technique has been applied to single degree of freedom systems only. Also, previously the control forces:: were represented in the limiting performance problem by piecewise constant bursts of forces (of a preselected time interval) over the time 
period of concern. This often results in a response that reverses its direction suddenly and repeatedly, thus making it difficult to identify parameters by curve fitting. In order to ease the task of parameter identification, we have reformulated the transient limiting performance problem using "smooth" control forces, i.e. ones represented by Fourier sertes. It is the purpose of this work to outline the formulation and to demonstrate the new methodology for multi-degree, multi-design element systems subject to transient disturbances.

\section{Limiting Performance Formulation}

Consider the problem of designing portions of a dynamic system subject to transient disturbances so that some index of performance is minimized (or maximized) and certain response constraints are satisfied. Replace these portions, which can be nonlinear in the state vaxiables, by control forces $u(t)$ and suppose that the remaining portions of the system are linear as are the overall kinematics. It is also assumed that both the performance index and constraints are linear functions of the state variables. For such systems, the limiting performance problem can be formulated as one of linear programming.

\subsection{Equations of Motion}

The general equations of motion for the system described above can be written in the form 


$$
\underline{M} \underline{\bar{x}}+\underline{C} \dot{x}+\underline{K} \bar{x}=\underline{V} \bar{u}+\underline{F} \bar{f}
$$

where

$$
\begin{aligned}
& \overline{\mathrm{x}}=\text { displacement vector } \\
& \overline{\mathrm{u}}=\text { control force vector which replaces portions of the system } \\
& \quad \text { being designed } \\
& \overline{\mathrm{f}}=\text { forcing function vector } \\
& \underline{M}=\mathrm{N} \times \mathrm{N} \text { mass matrix, } \mathrm{N} \text { being the number of degrees of freedom (DOF) } \\
& \underline{\mathrm{C}}=\mathrm{N} \times \mathrm{N} \text { damping matrix } \\
& \underline{\mathrm{K}}=\mathrm{N} \times \mathrm{N} \text { stiffness matrix } \\
& \underline{\mathrm{V}}=\mathrm{N} \times \mathrm{J} \text { coefficient matrix associated with control force } \\
& \quad \text { vector, J being the number of control forces } \\
& \underline{F}=\mathrm{N} \times \mathrm{L} \text { coefficient matrix associated with forcing function } \\
& \text { the smooth control forces lead to better designed systems. Each control } \\
& \text { force will be represented by a finite-term Fourier series of preselected } \\
& \text { wise constant control forces are employed. It will be demonstrated that } \\
& \text { functions, e.g. Fourfer series. Previously (Ref, } 1 \text { ) "nonsmooth" piece- } \\
& \text { We choose to represent the control forces in terms of "smooth" }
\end{aligned}
$$
basic frequency:

$$
\begin{aligned}
u_{j} & =\sum_{n=1}^{N F}\left(a_{j n} \cos \omega_{n} t+b_{j n} \sin \omega_{n} t\right)+a_{j 0}, j=1,2, \ldots, . J \\
\text { or } \bar{u} & =\sum_{n=1}^{N F}\left(\bar{A}_{n} \cos \omega_{n} t+\bar{B}_{n} \sin \omega_{n} t\right)+\bar{A}_{0}
\end{aligned}
$$


where

$$
\bar{A}_{n}=\left\{\begin{array}{c}
a_{1 n} \\
a_{2 n} \\
\vdots \\
a_{j n}
\end{array}\right\}, \quad \bar{B}_{n}=\left\{\begin{array}{c}
b_{1 n} \\
b_{2 n} \\
\vdots \\
\vdots \\
b_{j n}
\end{array}\right\}, \quad \bar{A}_{0}=\left\{\begin{array}{c}
{ }^{a} 10 \\
a_{20} \\
\vdots \\
a_{j 0}
\end{array}\right\}
$$

and $\omega_{n}=n \pi / T$, $T$ being the half period. Note that this representation of the control forces places no linearity restrictions on them.

Similarly, the relative displacements are represented by

$$
\begin{aligned}
x_{i} & =\sum_{n=1}^{N F}\left(p_{i n} \cos \omega_{n} t+q_{i n} \sin \omega_{n} t\right)+p_{i 0}, \quad i=1,2, \ldots, N \\
\text { or } \quad \bar{x} & =\sum_{n=1}^{N F}\left(\bar{P}_{n} \cos \omega_{n} t+\bar{Q}_{n} \sin \omega_{n} t\right)+\bar{p}_{0}
\end{aligned}
$$

where

$$
\mathrm{P}_{\mathrm{n}}=\left\{\begin{array}{c}
\mathrm{p}_{1 \mathrm{n}} \\
\mathrm{p}_{2 \mathrm{n}} \\
\vdots \\
\mathrm{p}_{\mathrm{Nn}}
\end{array}\right\}, \mathrm{Q}_{\mathrm{n}}=\left\{\begin{array}{c}
\mathrm{q}_{1 \mathrm{n}} \\
\mathrm{q}_{2 \mathrm{n}} \\
\vdots \\
\mathrm{q}_{\mathrm{Nn}}
\end{array}\right\} \text {, and } \mathrm{P}_{0}=\left\{\begin{array}{c}
\mathrm{p}_{10} \\
\mathrm{p}_{20} \\
\vdots \\
\mathrm{p}_{\mathrm{NO}}
\end{array}\right\}
$$

and,

$$
\bar{f}=\sum_{n}^{N F}\left(\bar{R}_{n} \cos \omega_{n} t+\bar{s}_{n} \sin \omega_{n} t\right)+\bar{R}_{0}
$$


with

$$
\overrightarrow{\mathrm{R}}_{\mathrm{n}}=\left\{\begin{array}{c}
\mathrm{r}_{\mathrm{In}} \\
\mathrm{r}_{2 \mathrm{n}} \\
\vdots \\
\mathrm{r}_{\mathrm{Ln}}
\end{array}\right\}, \quad \overline{\mathrm{s}}_{\mathrm{n}}=\left\{\begin{array}{c}
\mathrm{s}_{1 \mathrm{n}} \\
\mathrm{s}_{2 \mathrm{n}} \\
\vdots \\
\mathrm{s}_{\mathrm{Ln}}
\end{array}\right\} \quad \text {, and } \overline{\mathrm{R}}_{0}=\left\{\begin{array}{c}
\mathrm{r}_{10} \\
\mathrm{r}_{20} \\
\vdots \\
\mathrm{r}_{\mathrm{L} 0}
\end{array}\right\}
$$

$$
\begin{aligned}
& \text { Differentiation of (3) results in } \\
& \dot{\bar{x}}=\sum_{n}\left(-\omega_{n} \bar{P}_{n} \sin \omega_{n} t+\omega_{n} \bar{Q}_{n} \cos \omega_{n} t\right) \\
& \text { and } \quad \stackrel{\ddot{x}}{x}=\sum_{n}\left(-\omega_{n}^{2} \bar{P}_{n} \cos \omega_{n} t-\omega_{n}^{2} \bar{Q}_{n} \sin \omega_{n} t\right)
\end{aligned}
$$

Now substitute equations (2) - (6) into (1). This yields

$$
\begin{aligned}
& \sum_{n} \underline{M}\left(-\omega_{n}^{2} \bar{P}_{n} \cos \omega_{n} t-\omega_{n}^{2} \bar{Q}_{n} \sin \omega_{n} t\right)+\sum_{n} \underline{C}\left(-\omega_{n} \bar{P}_{n} \sin \omega_{n} t+\omega_{n} \bar{Q}_{n} \cos \omega_{n} t\right) \\
& +\sum_{n} \underline{K}\left(\bar{P}_{n} \cos \omega_{n} t+\bar{Q}_{n} \sin \omega_{n} t\right)+\underline{K} \bar{P}_{0}=\sum \underline{V}\left(\bar{A}_{n} \cos \omega_{n} t+\bar{B}_{n} \sin \omega_{n} t\right) \\
& +\underline{V} \bar{A}_{0}+\sum_{n} \underline{F}\left(\bar{R}_{n} \cos \omega_{n} t+\bar{S}_{n} \sin \omega_{n} t\right)+\underline{F} \bar{R}_{0}
\end{aligned}
$$

Collecting terms of $\cos \omega_{n} t, \sin \omega_{n} t$ and constants on both sides and making use of the orthogonality on $(-T, T)$, we obtain

$$
\begin{aligned}
& \left(-\omega_{n}^{2} \underline{M}+\underline{K}\right) \bar{P}_{n}+\omega_{n} C \bar{Q}_{n}=\underline{V A}_{n}+\underline{F} \bar{R}_{n} \\
& -\omega_{n} C \bar{P}_{n}+\left(-w_{n}^{2} M+\underline{K}\right) \bar{Q}_{n}=\underline{V B}_{n}+\underline{F} \bar{S}_{n} \\
& \underline{K}_{0}=\underline{V} \bar{A}_{0}+\underline{F} \bar{R}_{0}
\end{aligned}
$$

for $n=1,2, \ldots, N$. 
Eqns. (7) are recognized to be a set of simultaneous linear equations in $\overline{\mathrm{P}}_{\mathrm{n}}, \overline{\mathrm{Q}}_{\mathfrak{n}}$ and $\overline{\mathrm{P}}_{0}$, the right hand side being the Fourier coefficients of $\bar{u}$ and the forcing functions. Thus we have shown through Eq. (7) that the Fourier coefficients of $\bar{x}$ are related to those of $\bar{u}$. Frequently, e.g., in the case of many shock isolation systems, the equations of motion can be written as

$$
\underline{M \bar{x}}=\underline{\bar{v}}+\underline{F \bar{f}}
$$

For this special case, it is necessary that we express the relative displacement $\bar{x}$ in terms of the Fourfer coefficients of the $\bar{u}$ 's by direct integration of (8) instead of solving Eqn. (7). Thus, one integration of (8) gives

$$
\underline{M} \dot{\bar{x}}=\sum_{n}^{N F} \underline{V}\left(\frac{\sin \omega_{n} t}{\omega_{n}} \bar{A}_{n}-\frac{\cos \omega_{n} t}{\omega_{n}} \bar{B}_{n}\right)+\underline{V} \bar{A}_{0} t+\underline{F} \int \bar{f} d t+\bar{C}_{1},
$$

and after a second integration

$\underline{M \bar{x}}=\sum_{n}^{N F} \underline{V}\left(-\frac{\cos \omega_{n} t}{\omega_{n}^{2}} \bar{A}_{n}-\frac{\sin \omega_{n} t}{\omega_{n}^{2}} \bar{B}_{n}\right)+\underline{V A}_{0} \frac{t^{2}}{2}+\underline{E} \iint \bar{f}_{d t}+\bar{C}_{1} t+\bar{C}_{2}$,

where $\bar{c}_{1}$ and $\bar{C}_{2}$ are integration constants to be determined by the initial conditions.

\subsection{Limiting Performance}

With the state variables now expressed linearly in terms of the Fourier coefficients $\bar{A}_{0}, \bar{A}_{n}, \bar{B}_{n}$ of the control forces $\bar{u}(t)$, and $\bar{f}$ being a known quantity, it is a simple matter to express the performance index and the constraints linearly also in terms of these coefficients. 
The limiting performance problem then becomes:

Find the Fourier coefficients $\bar{A}_{0}, \bar{A}_{n}, \bar{B}_{n}$, where $n=1,2, \ldots ., N F$, such that the performance index

$$
\psi=\max _{i} \max _{t}\left|\phi_{i}\left(A_{0}, \bar{A}_{n}, \bar{B}_{n}, \bar{E}, t\right)\right|, i=1,2, . ., I
$$

is minimized subject to the constraints

$$
\varepsilon_{k}^{L} \leq_{k}\left(A_{0}, \bar{A}_{n}, \bar{B}_{n}, \bar{f}, t\right) \leq \varepsilon_{k}^{U}, k=1,2, \ldots, k
$$

where the $\phi_{1}$ 's are I response functions and $E_{k}^{U}, \varepsilon_{k}^{L}$ are the upper and lower bounds of the $k^{\text {th }}$ constraint $\delta_{k}$. This problem is readily recognized as one of linear programing (LP) and it can easily be reduced to the standard LP form (Ref. 1). Then any available LP codes can be used to determine these coefficients and hence the optimal responses $u^{*}(t)$, $\overline{\mathrm{x}}^{*}(\mathrm{t}), \dot{\bar{x}}^{*}(\mathrm{t}), \ddot{\bar{x}}^{*}(t)$.

\section{Results}

The new design approach with "smooth" control force representation has been applied to a number of transient systems. Two selected examples are presented below.

\subsection{Two Degree of Freedon System}

Consider the two degree of freedom system shown in Fig. I(a). A step velocity of $\mathrm{V}_{0}=1 \mathrm{in} / \mathrm{sec}$ is applied to the rigld base over a period of $1.5 \mathrm{sec}$. The design problem is to determine the $k_{1}, k_{2}, c_{1}$ and $c_{2}$ so that the peak acceleration of $\mathrm{m}_{1}$ is minimized subject to the constraints that the rattlespaces $x_{1}$ and $x_{2}$ be 1 ess than $3 / 4$ in. 


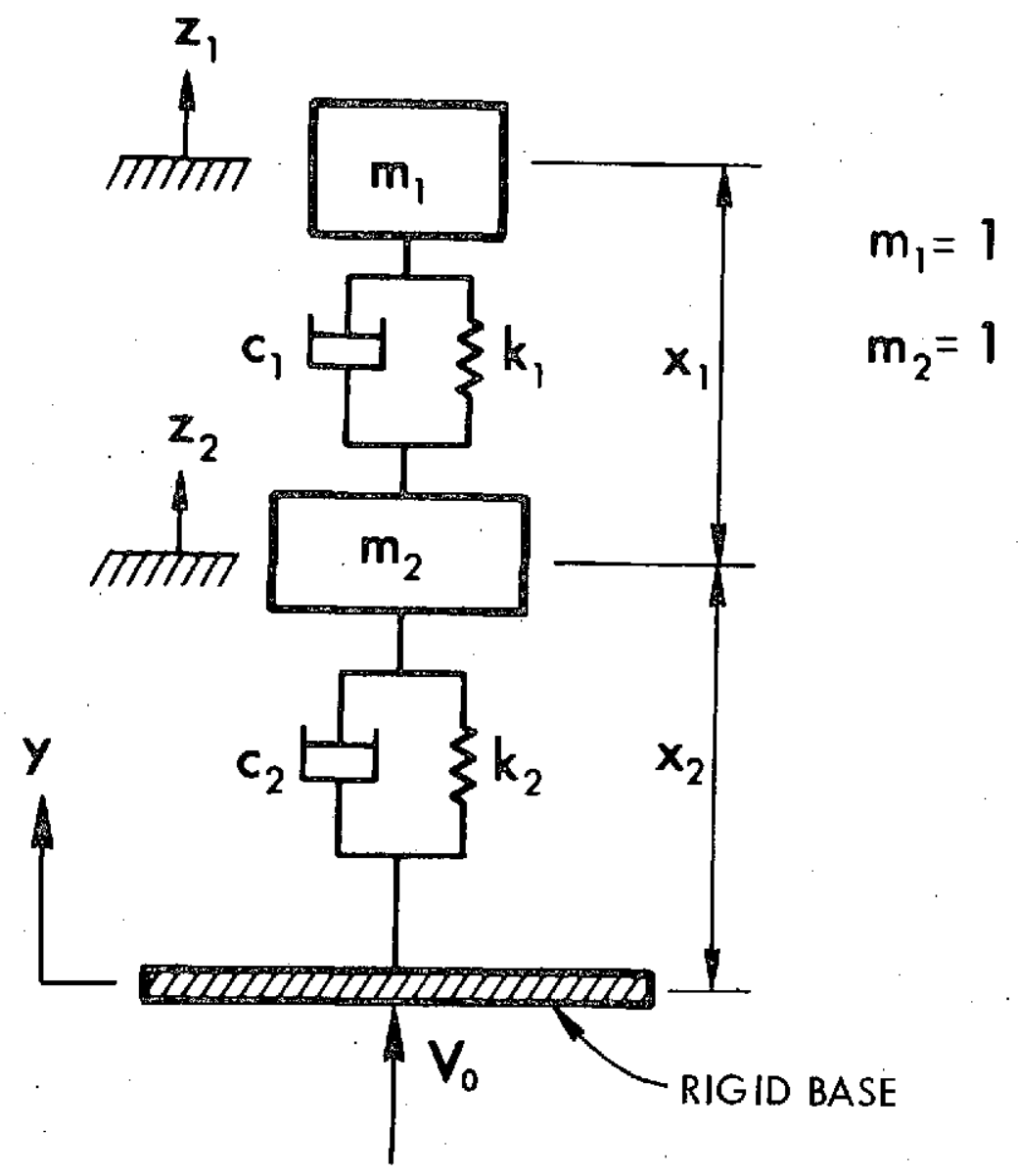

(a)

Fig. 1 (a) Two degree of freedom system.

Initial conditions:

$z_{1}(0)=z_{2}(0)=\dot{z}_{1}(0)=\dot{z}_{2}(0)=0$.

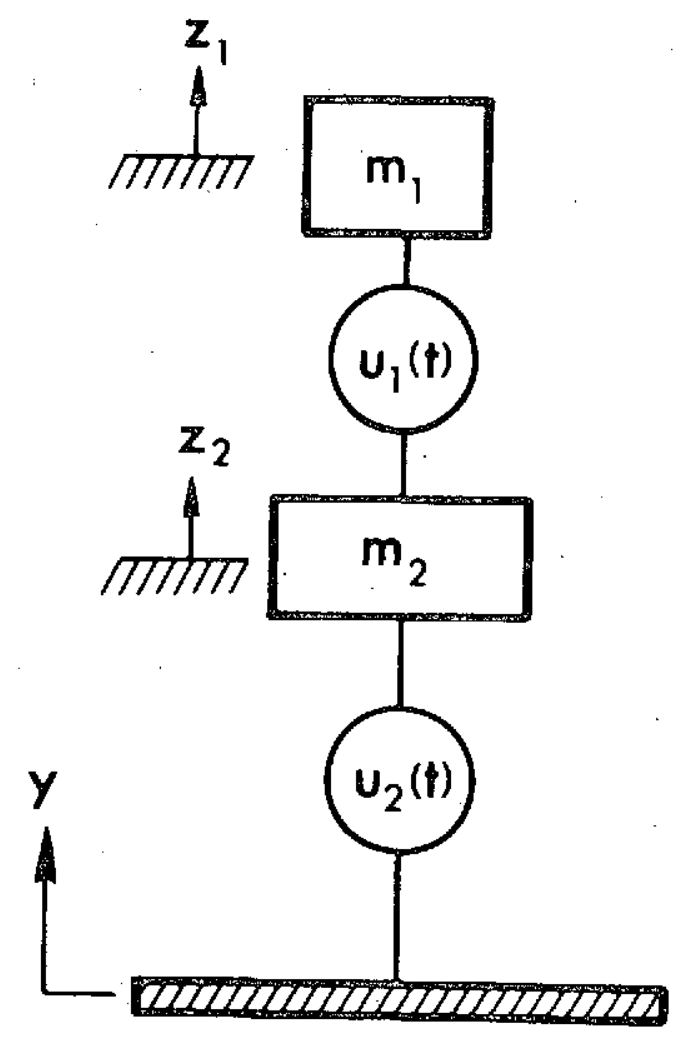

(b) Fig. 1 (b) System with isolators replaced by
control forces. 
The substitute system for the limiting performance study is shown in Fig. l(b). The governing equations are

$$
\begin{aligned}
& \ddot{z}_{1}+u_{1}=0 \\
& \ddot{z}_{2}-u_{1}+u_{2}=0
\end{aligned}
$$

with the kinematic conditions

$$
\begin{aligned}
& x_{2}=z_{2}-y \\
& x_{1}=z_{1}-z_{2}
\end{aligned}
$$

The limiting performance problem then is to minimize

$$
\psi=\max \left|\ddot{z}_{1}\right|
$$

with the constraints

$$
\begin{aligned}
& \left|x_{1}\right| \leq 3 / 4 \\
& \left|x_{2}\right| \leq 3 / 4
\end{aligned}
$$

The limiting performance solutions obtained by a linear programming technique are shown in Fig. 2 and 3 together with the results of parameter identification obtained by fitting $u_{1}^{*}(t)$ and $u_{2}^{*}(t)$ respectively to $k_{1} x_{1}^{*}(t)+c_{1} \dot{x}_{1}^{*}(t)$ and $k_{2} x_{2}(t)+c_{2} \dot{x}^{*}(t)$.

Here for simplicity, an ordinary unconstrained least square technique is used. The curves labeled "parameter identification" are the solutions to the equations of motion of the designed system. The limiting performance solution yields a peak acceleration of 1.35 for 


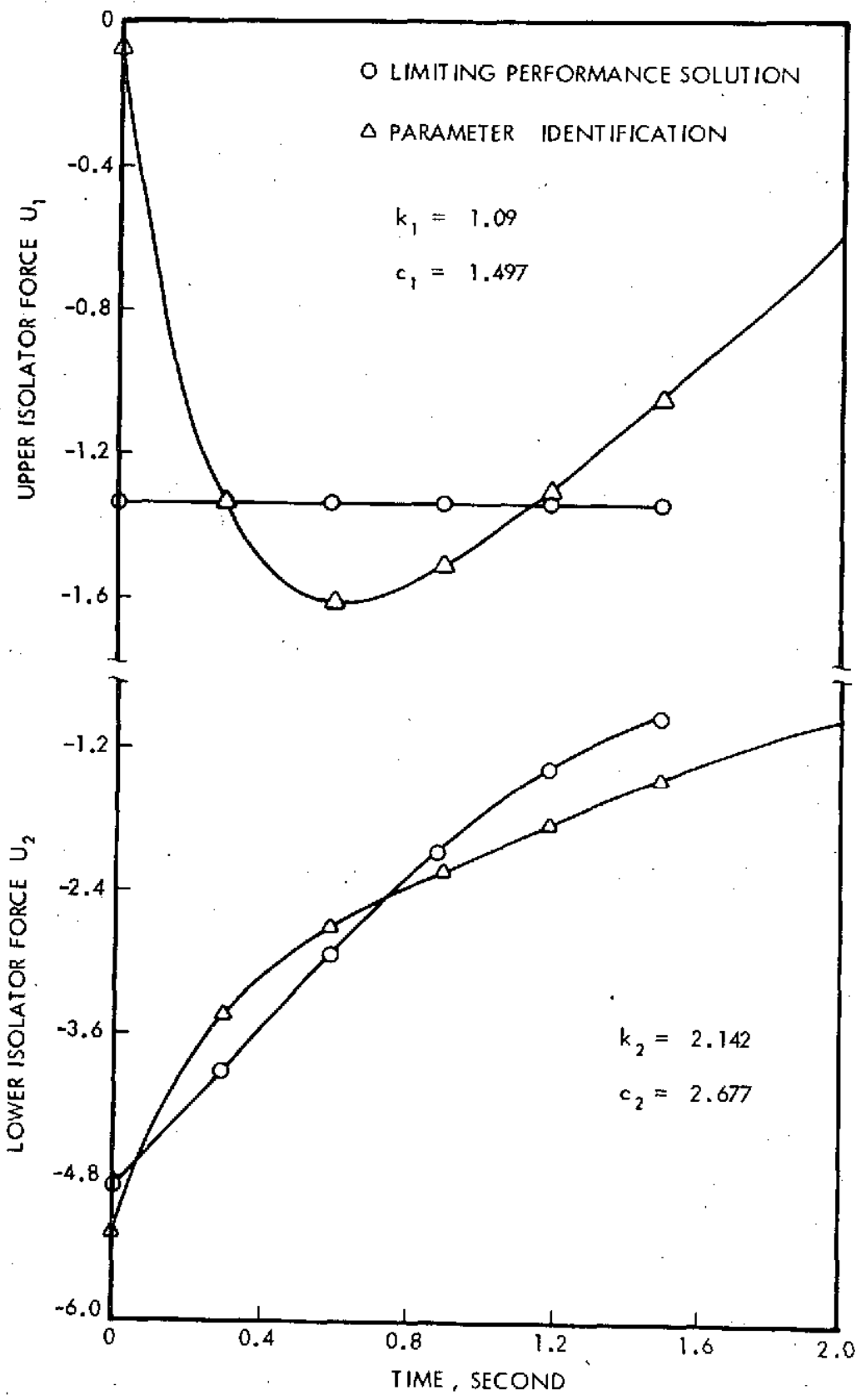

Fig. 2 Upper and lower Isolator response histories - force-time (by Fourier representation). 


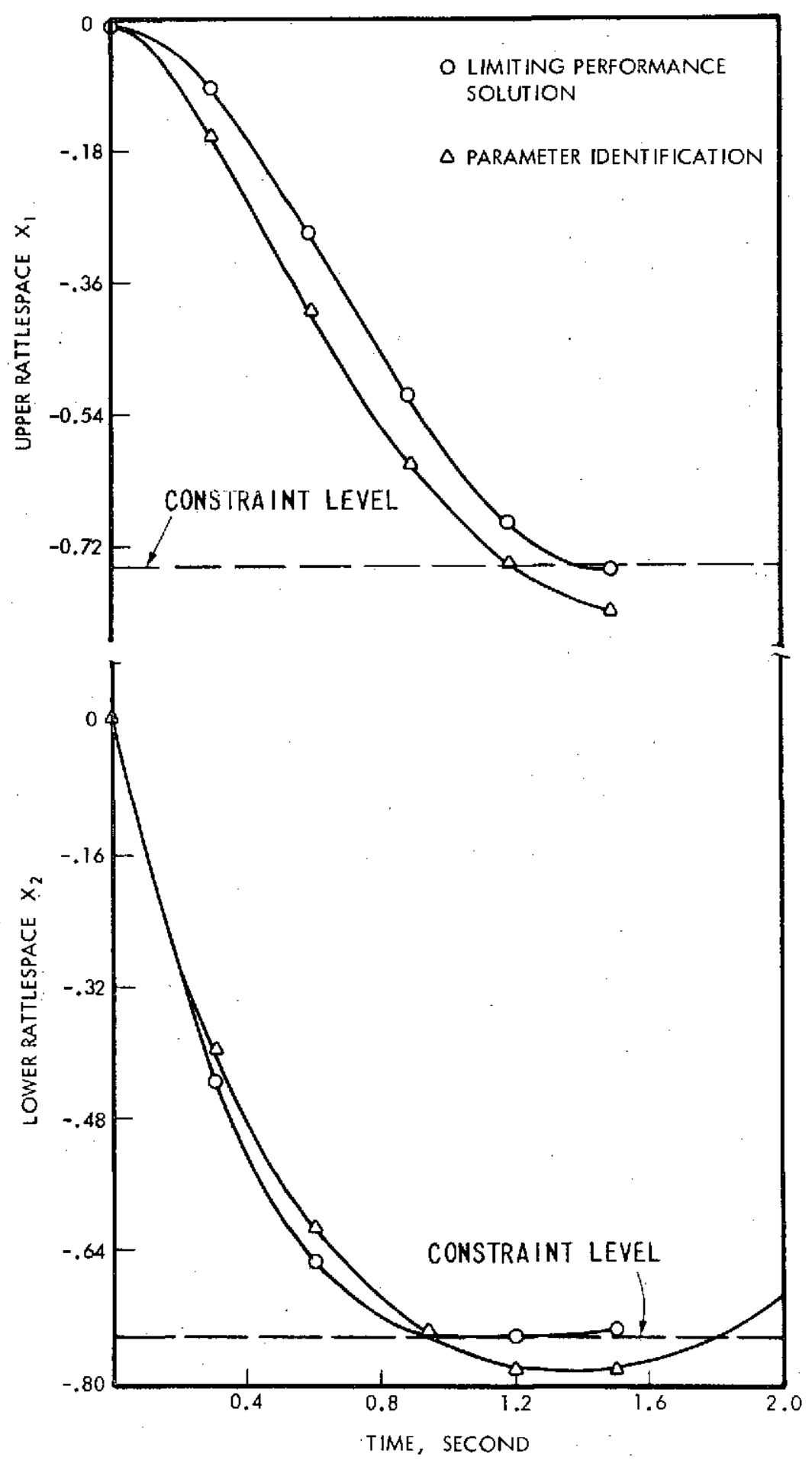

Fig. 3 Upper and lower isolator response histories - displacementtime (by Fourier representation). 
$m_{1}$ while the designed system gives 1.60 . It is also seen that the rattlespaces are slightly violated, by $8 \%$ for the upper one and $5.5 \%$ for the lower.

For purposes of comparison, the same problem was solved using the piecewise constant representation of the control forces (Ref. 1). The results are shown in Fig. 4 and 5 . It is apparent that although the limiting performance characteristic of $\max \left|\ddot{z}_{1}\right|=\max \left|u_{1}\right|$ are almost identical for the two representations, the task of curve fitting the isolator responses in this case (Fig. $4(\mathrm{~b})$ and $5(\mathrm{~b})$ ) is much more difficult than the case of "smooth" representation. Here the worst rattlespace (upper) violation is as much as $100 \%$ and the performance index is also considerably higher than the "smooth" method.

\subsection{Six Parameter Designs of Three Degree of Freedom System}

In order to make quantitative comparisons of the new design approach with conventional computer optimization, the structure of Fig. $6(a)$ was studied. The goal is to find the parameters $k_{1}, k_{2}, k_{3}, c_{1}, c_{2}, c_{3}$ such that for base inputs $y=t^{2} e^{-t}$ the maximum of the peak values of $\left|\ddot{z}_{1}\right|,\left|\ddot{z}_{2}\right|,|\ddot{\theta}|$ is minimized subject to

$$
\begin{array}{ll}
\left|x_{1}(t)+a \theta(t)\right| \leq \varepsilon_{1}, & \left|x_{1}(t)-e \theta(t)\right| \leq \varepsilon_{1} \\
\left|x_{2}(t)-b \theta(t)\right| \leq \varepsilon_{2}, & \left|x_{2}(t)+d \theta(t)\right| \leq \varepsilon_{2} .
\end{array}
$$



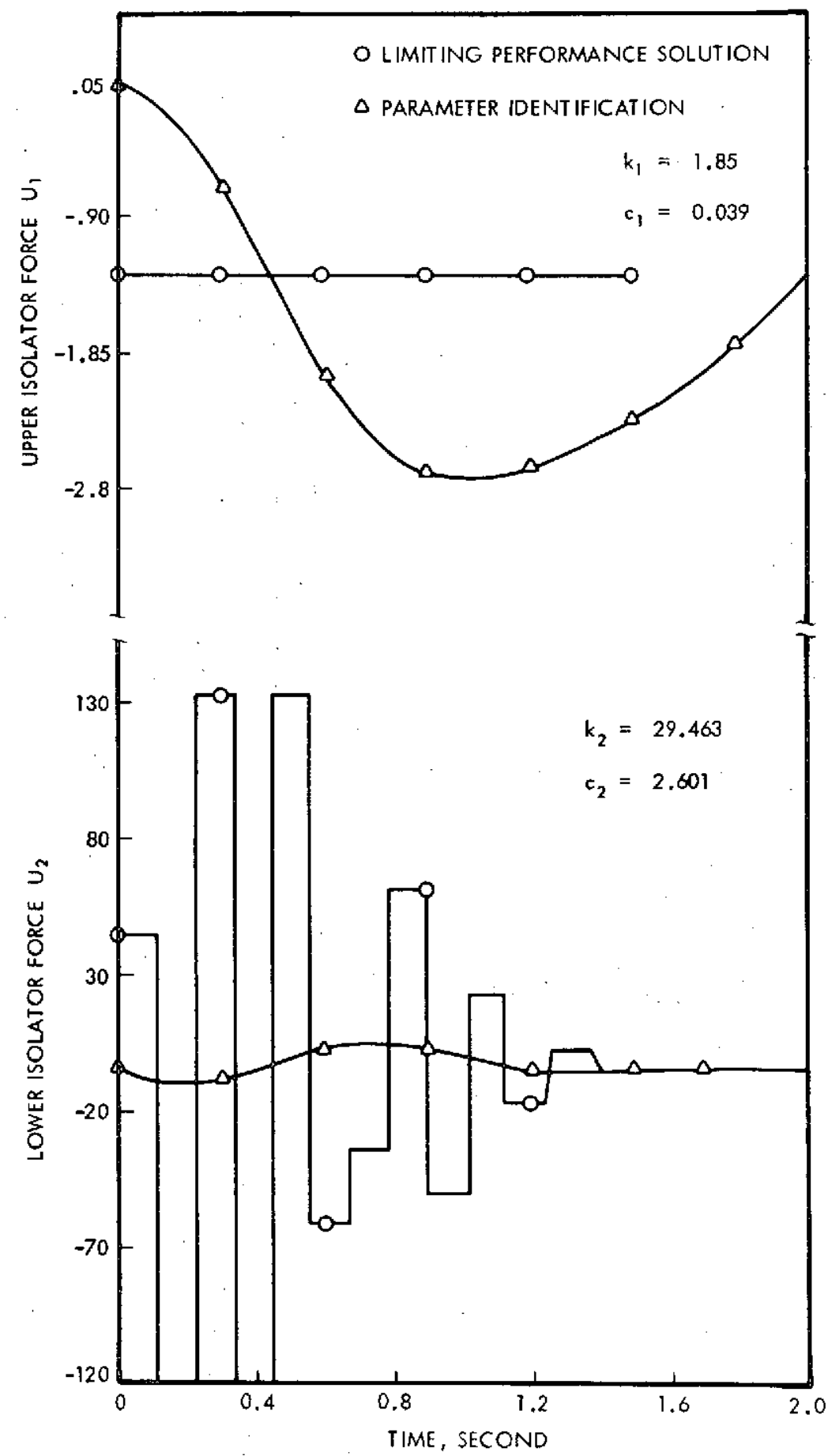

Fig. 4 Upper and lower isolator response histories - force-time (by piecewise constant representation). 


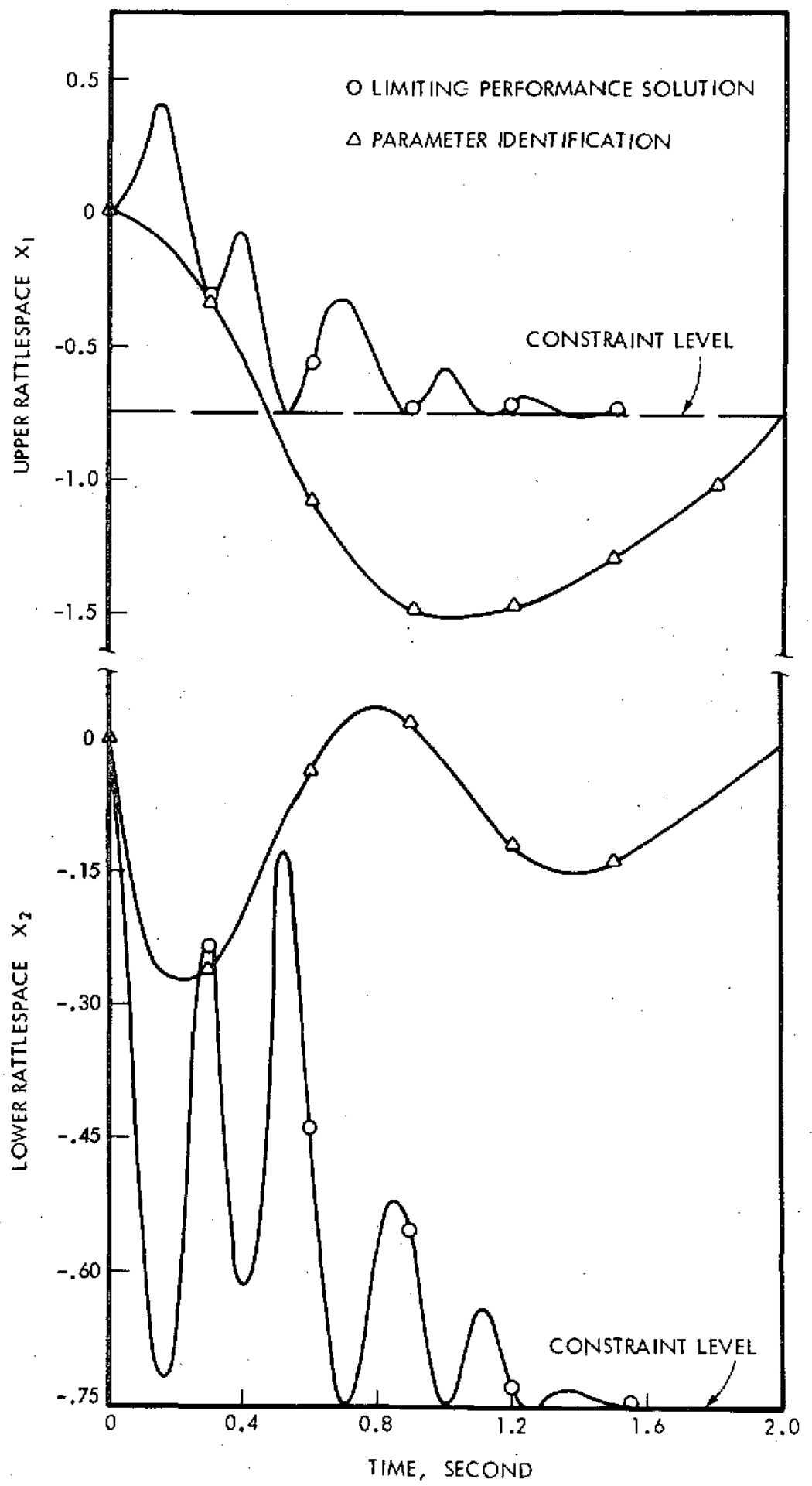

Fig. 5 Upper and lower isolator response histories - displacementtime (by piecewise constant representation). 


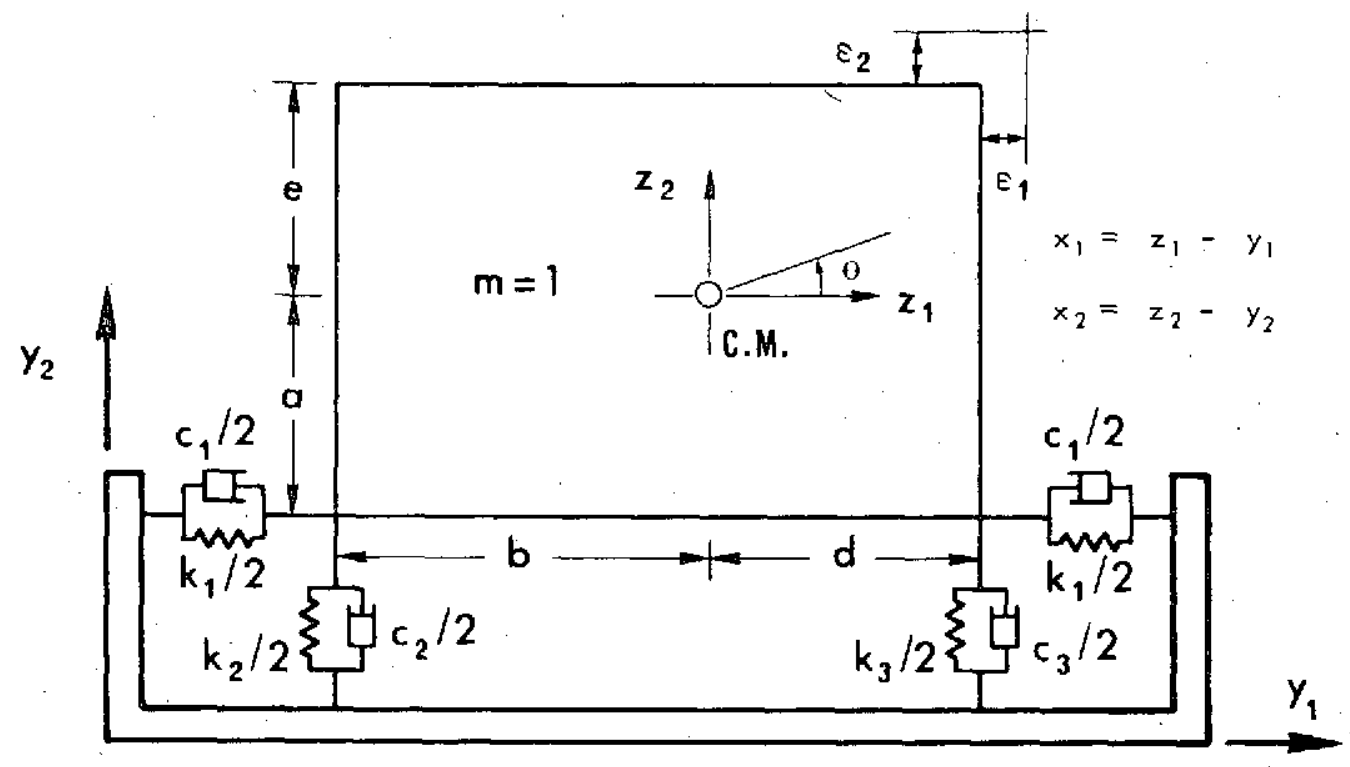

(a)

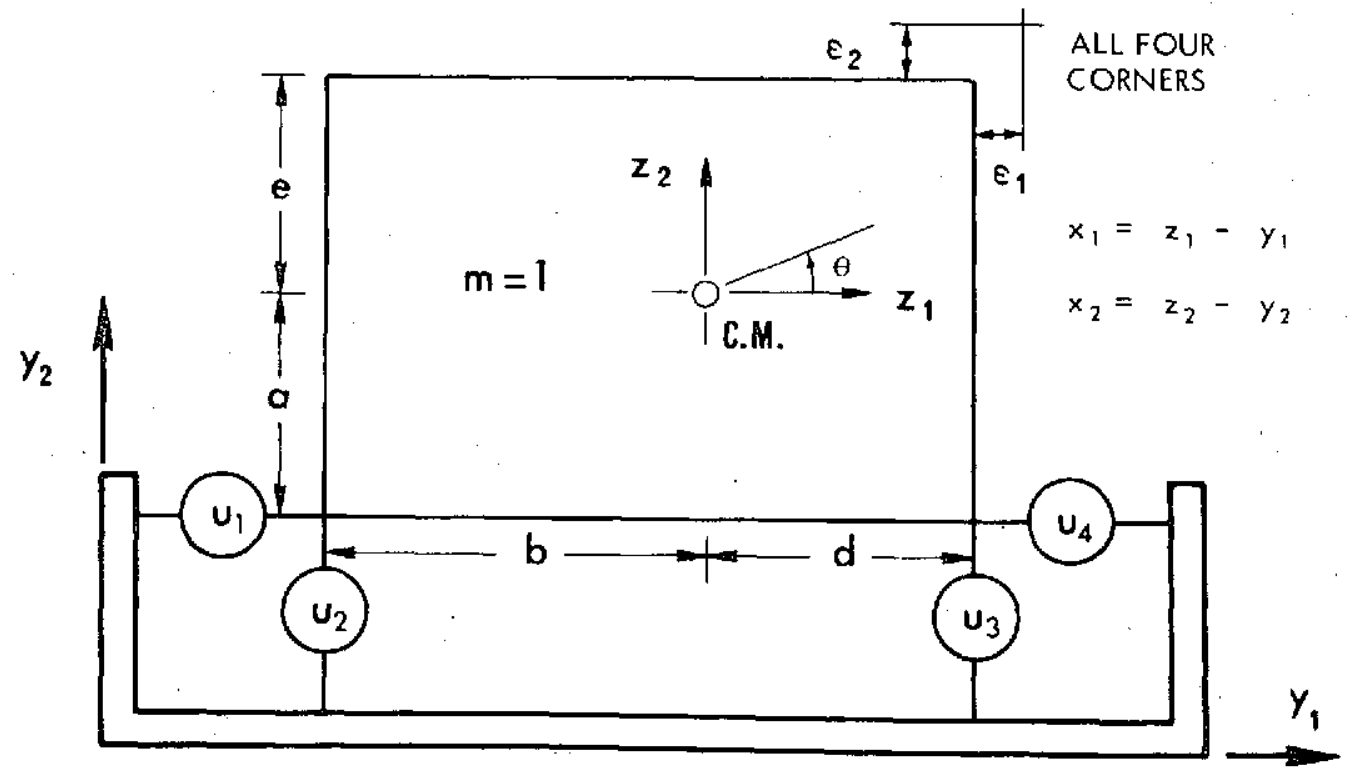

(b)

Fig. 6 (a) Three degree of freedom system. Initial conditions: $z_{1}(0)=z_{2}(0)=\theta(0)=\dot{z}_{1}(0)=\dot{z}_{2}(0)=\dot{\theta}(0)=0$.

(b) Substitute system used for limiting performance study. 
The substitute structure employed in the design process is shown in

Fig. 6(b). The equations of motion are

$$
\begin{aligned}
& \ddot{z}=-\left(u_{1}+u_{4}\right) \\
& \ddot{z}_{2}=-\left(u_{2}+u_{3}\right) \\
& \ddot{\theta}=-\frac{a}{\rho^{2}}\left(u_{1}+u_{4}\right)+\frac{b}{\rho^{2}} u-\frac{d}{\rho^{2}} u_{3},
\end{aligned}
$$

with the kinematic relations

$$
x_{1}=z_{1}-y_{1}, x_{2}=z_{2}-y_{2}
$$

and the initial conditions

$$
\dot{z}_{1}(0)=z_{2}(0)=\dot{z}_{1}(0)=\dot{z}_{2}(0)=0
$$

The radius of gyration is designated by $\rho$.

The design parameters are determined by matching the ideal isolator forces $u_{i}^{*}(t), i=1,2,3$, to

$$
\begin{aligned}
& g_{1}=\frac{k_{2}}{2}\left(x_{2}^{*}-b \theta^{*}\right)+\frac{c_{2}}{2}\left(\dot{x}_{2}^{*}-b \dot{\theta}^{*}\right) \\
& g_{2}=\frac{k_{3}}{2}\left(x_{2}^{*}+d \theta^{*}\right)+\frac{c_{3}}{2}\left(\dot{x}_{2}^{*}+d \dot{\theta}^{*}\right) \\
& g_{3}=k_{1}\left(x_{1}^{*}+a \theta^{*}\right)+c_{1}\left(\dot{x}_{1}^{*}+a \dot{\theta}^{*}\right),
\end{aligned}
$$

For $a=b=d=25, \quad \rho=23.5, \quad \varepsilon_{1}=\varepsilon_{2}=0.1$, a least square curve fitting technique leads to $\mathrm{k}_{1}=2.994, \mathrm{k}_{2}=7.129, \mathrm{k}_{3}=1.626, \mathrm{c}_{1}=2.716$, $c_{2}=5.062$ and $c_{3}=0.426$. The active objective functions are plotted in Fig. 7 together with the ideal isolator forces. It is seen that the peak objective function is 0.83 for the designed system, compared to the value of 0.6 given by the limiting performance solution. The constraint 


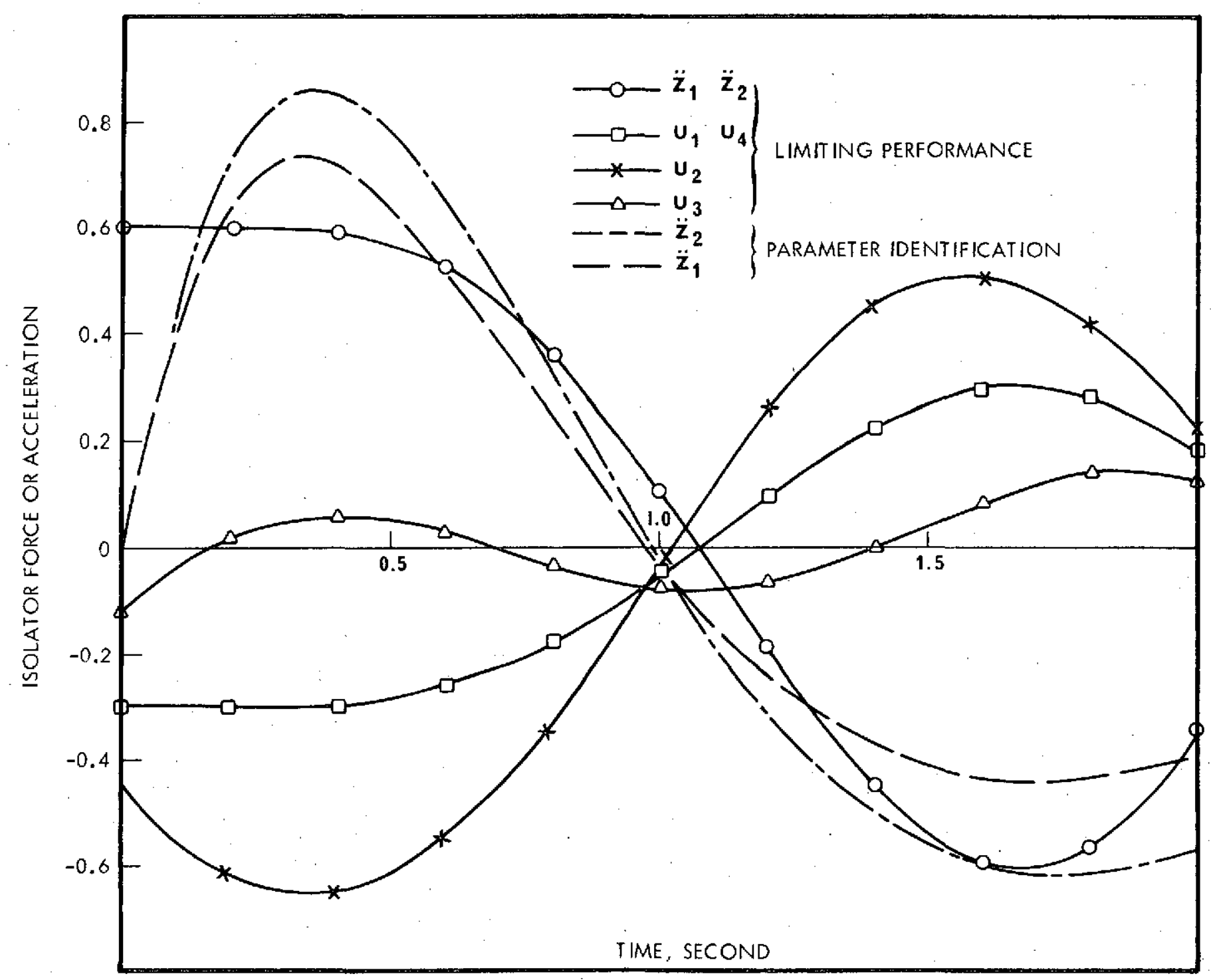

Fig. 7 Isolator response histories - force-time. 


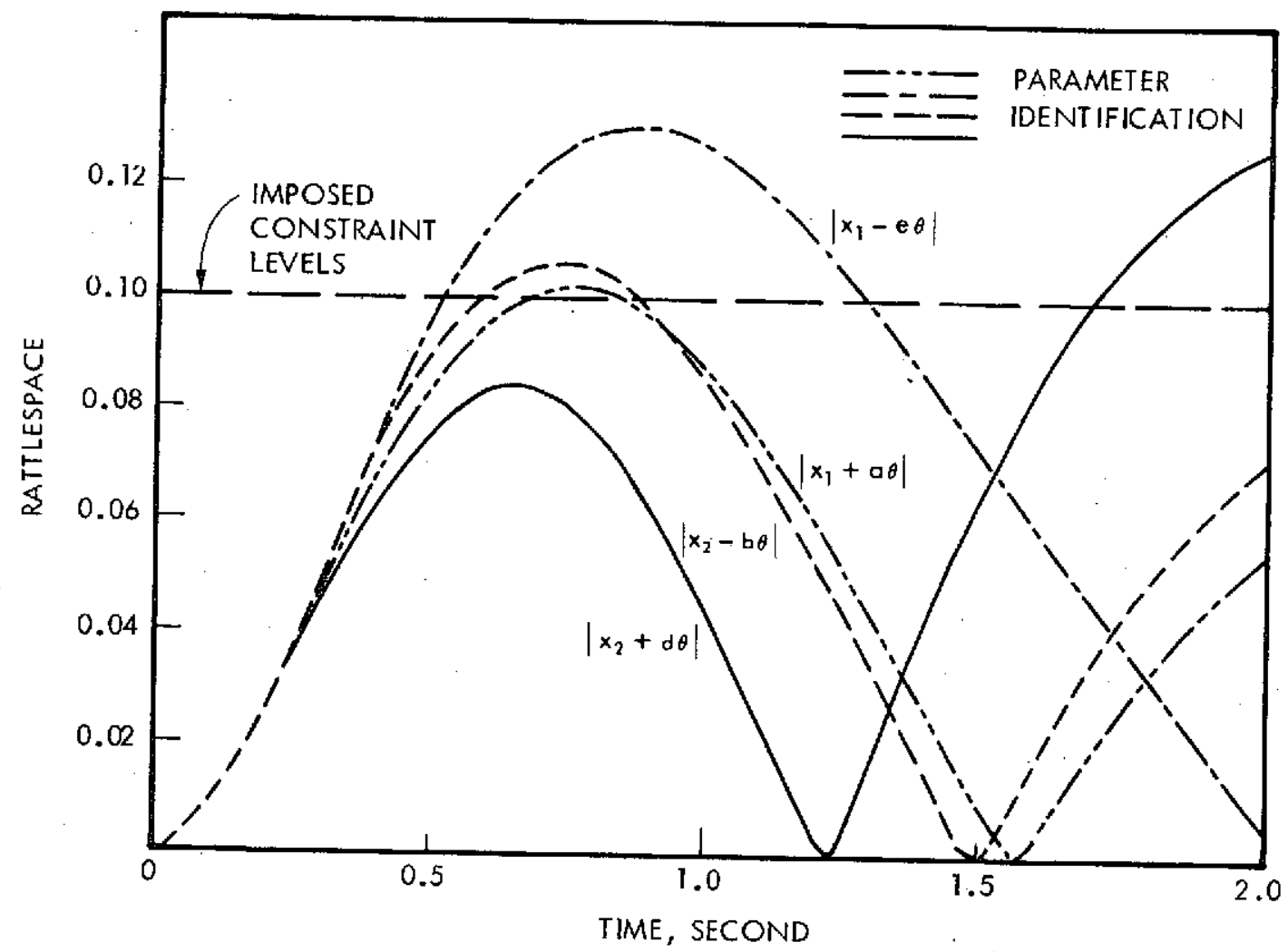

Fig. 8 Constraint responses for the designed system. 
responses are plotted in Fig. 8. The worst violation of the constraints is about $25 \%$. For the limiting performance study we chose $N F=2$ in the Fourier expansion and a half period of two seconds was selected. This is close to the time at which the base velocity drops to zero and its displacement reaches its maximum.

This particular design problem was chosen because the same system was designed using classical techniques in Ref. 4. There the publicly available (ASIAC, Ref. 5) optimization program POSI was employed. This standard computational optimization program selected a system with an optimal objective function of 1.15 versus our 0.83 . Our more optimal design, of course, is partly a result of constraint violations. This apparent problem, which is considered below, was suggested in Ref. 3 as being so serious as to make questionable the value of this method.

Perhaps the most interesting result is the comparison of computer times. Typical designs (such as the one just presented) made by the new approach took about one to two percent of the computer processor time expended by the classical computer optimization using POSI.

The problem of constraint violation is not as serious as it may seem. Techniques more sophisticated than unconstrained least square fits, e.g., a constrained min-max fit, may be used to reduce the constraint violations. Constrained parameter identffication techniques are also useful if it is known that for practical consideration the design parameters must be restricted to certain values. In addition, it is possible to correct constraint violations by finding the design parameters 
based on matching the response being violated (Ref. 1). Also, this problem can be remedied by using our optimal design parameters as starting values in a conventional computer optimization program. For the above problem a design that satisfied all constraints was obtained after one iteration by POSI.

4. Conclusions

It has been demonstrated that the indirect synthesis design approach can be used to obtain a near optimal design for multi-degree of freedom and multi-design-element systems - at least for the inputs considered here. The design occasionally violates the desired constraint levels. As explained, however, corrective measures can be taken. 
References

1. Sevin, E., and Pilkey, W. D., Optimum Shock and Vibration Isolation, Monograph No. 6, The Shock and Vibration Information Center, Washington, 1971.

2. Afimiwala, K. A., and Mayne, R. W., "Optimum Design of an Impact Absorber," Trans. ASME, Journal of Engineering for Industry, Vo1. 96, 1974.

3. Metwalli, S. M., Afimiwala, K. A., and Mayne, R. W., "Optimum Design of Vehicle Suspensions," ASME Paper No. 73-ICT-58, 1973.

4. Klein, G. H., Axelband, E. I., and Parker, R. E., "Optimum Shock Isolation for Underground Protective Structure," ARWL-TR-69-178, Sept. 1970 .

5. Aerospace Structures Information and Analysis Center, AFFDL/FBR, Wright-Patterson AFB, Ohio 45433, 


\section{UNIVERSITY OF VIRGINIA}

\section{School of Engineering and Applied Science}

The University of Virginia's School of Engineering and Applied Science has an undergraduate enrollment of approximately 1,000 students with a graduate enrollment of 350 . There are approximately 120 faculty members, of whom, about $90 \%$ hold a doctorate. Excellence in graduate education is aided and supplemented by a research program approximating $\$ 3$ million per year. This relatively high level of participation in sponsored research is one factor which helps our faculty consistently to maintain high quality graduate education at all degree levels.

As research is an integral part of the educational program, research interests parallel academic specialities. These interests range from the traditional engineering departments of Chemical, Civil, Electrical and Mechanical to include departments of Biomedical Engineering, Engineering Science \& Systems, Materials Science, Nuclear Engineering, and Applied Mathematics \& Computer Science. In addition to these departmental interests, there are interdepartmental groups in the areas of Automatic Controls and Applied Mechanics. All departments are authorized to offer the doctorate while the Biomedical and Materials Science Departments are graduate degree granting departments only.

The School of Engineering and Applied Science, is an integral part of an outstanding University, which has strong professional Schools of Law, Medicine, and Business Administration. In addition, the College of Arts and Sciences has strong basic science departments in Mathematics, Physics, Chemistry, and other departments relevant to the engineering research program. This not only provides an excellent scholarly climate, but also enhances the school's potential for creating truly interdisciplinary teams in the pursuit of our basic goals of education, research, and public service.

Inside this cover are listed some of the present research activities of the department from which this report originates. For more information on this or other areas, address the department chairman or Dean J. E. Gibson, Commonwealth Professor and Dean, School of Engineering and Applied Science, University of Virginia, Charlottesville, Virginia 22901. 